\title{
NUMERICAL TRIVIALITY AND PULLBACKS
}

\author{
BRIAN LEHMANN
}

Abstract. Let $f: X \rightarrow Z$ be a surjective morphism of smooth complex projective varieties with connected fibers. Suppose that $L$ is a pseudoeffective divisor that is $f$-numerically trivial. We show that there is a divisor $D$ on $Z$ such that $L \equiv f^{*} D$.

\section{INTRODUCTION}

We consider the following question:

Question 1.1. Let $f: X \rightarrow Z$ be a surjective morphism of smooth complex projective varieties with connected fibers. Suppose that $L$ is a pseudoeffective $\mathbb{R}$-Cartier divisor that is numerically trivial on the (general) fibers of $f$. Is $L$ numerically equivalent to the pull-back of a divisor on $Z$ ?

When $L$ is not pseudo-effective the answer is an emphatic "no" (see Examples 4.1 and 5.1). Thus it is perhaps surprising that there is a positive answer for pseudo-effective divisors. The most restrictive situation is to ask that $L$ be numerically trivial on every fiber of $f$. In this case $L$ is actually numerically equivalent to the pullback of a divisor on $Z$ :

Theorem 1.2. Let $f: X \rightarrow Z$ be a surjective morphism with connected fibers from a normal complex projective variety $X$ to a $\mathbb{Q}$-factorial normal complex projective variety $Z$. Suppose that $L$ is a pseudo-effective $\mathbb{R}$-Cartier divisor. Then $L$ is $f$-numerically trivial if and only if there is a pseudoeffective $\mathbb{R}$-Cartier divisor $D$ on $Z$ such that $L \equiv f^{*} D$.

Again, the pseudo-effectiveness of $L$ is crucial: the dimension of the space of divisors that are $f$-numerically trivial will generally be larger than the dimension of $N^{1}(Z)$.

For applications it is more useful to require that $L$ be numerically trivial only on a general fiber of $f$. To handle this case we need a systematic way of discounting the non-trivial behavior along special fibers. For surfaces the behavior of special fibers is captured by the Zariski decomposition. The analogous construction in higher dimensions is the divisorial Zarkiski decomposition of [Nak04]. Given a pseudo-effective $\mathbb{R}$-Cartier divisor $L$, the divisorial Zariski decomposition

$$
L=P_{\sigma}(L)+N_{\sigma}(L)
$$

2010 Mathematics Subject Classification. Primary 14C20.

The author is supported by NSF Award 1004363. 
expresses $L$ as the sum of a "movable part" $P_{\sigma}(L)$ and a "fixed part" $N_{\sigma}(L)$ (see Definition 2.8).

Theorem 1.3. Let $f: X \rightarrow Z$ be a surjective morphism with connected fibers from a normal complex projective variety $X$ to an integral complex projective variety $Z$. Suppose that $L$ is a pseudo-effective $\mathbb{R}$-Cartier divisor such that $\left.L\right|_{F} \equiv 0$ for a general fiber $F$ of $f$. Then there is a smooth birational model $\phi: Y \rightarrow X$, a map $g: Y \rightarrow Z^{\prime}$ birationally equivalent to $f$, and a pseudo-effective $\mathbb{R}$-Cartier divisor $D$ on $Z^{\prime}$ such that $P_{\sigma}\left(\phi^{*} L\right) \equiv$ $P_{\sigma}\left(g^{*} D\right)$.

The conclusion of Theorem 1.3 also holds if $L$ only satisfies the weaker requirement $\left.P_{\sigma}(L)\right|_{F} \equiv 0$ for a general fiber $F$.

In the setting of Theorems 1.2 and 1.3 the birational invariants of $L$ and $D$ coincide:

- $\kappa(D)=\kappa(L)$, where $\kappa$ denotes the Iitaka dimension.

- $\nu(D)=\nu(L)$, where $\nu$ denotes the numerical dimension of [Nak04] and [Bou04].

Indeed, both invariants are unchanged by passing to the positive part $P_{\sigma}$ and by surjective pullbacks between normal varieties. For the Iitaka dimension, the first property follows immediately from the definition and the second is verified by [Nak04] II.3.11 Lemma. For the numerical dimension, the first property is verified by [Leh13] Theorem 6.7. We prove the second:

Proposition 1.4. Let $f: X \rightarrow Z$ be a surjective morphism of integral complex projective varieties. Let $D$ be a pseudo-effective $\mathbb{R}$-Cartier divisor on $Z$. Then $\nu(D)=\nu\left(f^{*} D\right)$.

The analogue of Question 1.1 for $\mathbb{Q}$-linear equivalence is well understood, with the most general statements due to Nakayama. Theorem 1.3 is the numerical version of [Nak04] V.2.26 Corollary, and in particular [Nak04] gives stronger results when either $\kappa(L) \geq 0$ or when $f_{*} \mathcal{O}_{X}(L) \neq 0$.

To apply Theorems 1.2 and 1.3, one must find a morphism $f: X \rightarrow Z$ such that $L$ is numerically trivial along the fibers. [BCE $\left.{ }^{+} 02\right]$, [Eck05], and [Leh14] show that such maps can be constructed by taking the quotient of $X$ by subvarieties along which $L$ is numerically trivial. In fact, there is a maximal fibration such that $L$ is numerically trivial (properly interpreted) along the fibers. Thus Theorems 1.2 and 1.3 pair naturally with the reduction map theory developed in these papers.

Remark 1.5. Suppose given a morphism $\pi: Y \rightarrow X$ of smooth projective varieties. The recent paper [DJV13] analyzes the kernel of the pushforward map $\pi_{*}$ on numerical classes of Weil divisors. In particular, they show that any pseudo-effective divisor class in the kernel of $\pi_{*}$ is a limit of classes of effective divisors contracted by $\pi$. Theorem 1.3 is a stronger statement, and yields a short proof of [DJV13, Theorem 5.7]. The techniques can also be used for cycles of higher codimension; this will be demonstrated in upcoming work. 
Remark 1.6. The proofs of Theorems 1.2 and 1.3 are similar in spirit to the work of $\left[\mathrm{BCE}^{+} 02\right]$. In the course of the proof of $\left[\mathrm{BCE}^{+} 02\right.$, Theorem 2.1], the authors show that if a nef divisor is numerically trivial on the fibers of a map with connected fibers, and also numerically trivial along a multisection, then the nef divisor is numerically trivial. This is a very special case of Theorem 1.2 .

1.1. Outline. Nakayama's work on the divisorial Zariski decomposition is the key tool for the statements analogous to Theorems 1.2 and 1.3 in the context of $\mathbb{Q}$-linear equivalence. Nakayama's ideas also play an important role in the proof of Theorem 1.3. In Section 2, we review Nakayama's theory with some slight modifications for the numerical setting.

However, there seems to be no way to directly adapt the arguments of $[\mathrm{Nak04}]$ to our situation. Note that the statements involving $\mathbb{Q}$-linear equivalence hold for non-pseudo-effective divisors as well as pseudo-effective ones, whereas our main theorems do not.

Our main contribution is to find another way to construct a "candidate" divisor $D$ on the base of the map. We achieve this by cutting down to the case when $f$ is generically finite. A key conceptual point is that a numerical class is determined by intersections against curves not contained in a fixed countable union of codimension 2 subvarieties.

Section 2 reviews the numerical versions of ideas of [Nak04]. Section 3 gathers some technical results on generically finite maps. Sections 4 and 5 prove Theorems 1.2 and 1.3 respectively.

I thank B. Bhatt, A.M. Fulger, and Y. Gongyo for some helpful conversations.

\section{Preliminaries}

We work over the base field $\mathbb{C}$. All varieties are irreducible and reduced. A divisor will always mean an $\mathbb{R}$-Cartier divisor unless otherwise qualified.

2.1. Notation. We will use the standard notations $\sim, \sim_{\mathbb{Q}}, \sim_{\mathbb{R}}$, and $\equiv$ to denote respectively linear equivalence, $\mathbb{Q}$-linear equivalence, $\mathbb{R}$-linear equivalence, and numerical equivalence of divisors.

Definition 2.1. Suppose that $f: X \rightarrow Z$ is a morphism of integral projective varieties. We say that

- A curve $C$ on $X$ (or a curve class $\left.\alpha \in N_{1}(X)\right)$ is $f$-vertical if it has vanishing intersection against $f^{*} H$ for some ample Cartier divisor $H$ on $Z$.

- An $\mathbb{R}$-Cartier divisor $L$ on $X$ is $f$-numerically trivial if it has vanishing intersection with every $f$-vertical curve class.

Suppose that $f: X \rightarrow Z$ is a surjective morphism of integral projective varieties. Using generic flatness and the closedness of the Hilbert scheme, one sees that there is a birational model $\psi: T \rightarrow Z$ such that for the main 
component $W$ of $X \times_{Z} T$ the induced map $g: W \rightarrow T$ is flat. We say that $g: W \rightarrow T$ is a flattening of $f$.

2.2. Surfaces. We begin by considering Question 1.1 for surfaces. For surfaces, the Zariski decomposition is the key tool.

Theorem 2.2 ([Zar64],[Fuj79]). Let $S$ be a smooth projective surface and let $L$ be a pseudo-effective $\mathbb{R}$-Cartier divisor on $S$. There is a unique decomposition

$$
L=P+N
$$

where $P$ is a nef divisor and $N$ is an effective divisor satisfying

(1) $P \cdot N=0$.

(2) If $N \neq 0$, the intersection matrix defined by the components of $N$ is negative definite.

We will use this theorem to describe how a divisor $L$ behaves along special fibers of an $L$-trivial morphism. Although all the following lemmas are wellknown, it seems worthwhile to repeat the proofs here.

Lemma 2.3. Let $f: S \rightarrow C$ be a surjective morphism with connected fibers from a smooth projective surface $S$ to a smooth projective curve $C$. Let $L$ be a pseudo-effective $\mathbb{R}$-Cartier divisor on $S$ such that $L \cdot F=0$ for a general fiber $F$ of $f$.

(1) If $L \cdot D=0$ for every $f$-vertical curve $D$, then $L$ is nef.

(2) If $L \cdot D \neq 0$ for some $f$-vertical curve $D$ contained in a fiber $F_{0}$, then there is an $f$-vertical curve $G$ contained in $F_{0}$ satisfying $L \cdot G<0$.

Proof. Let $L=P+N$ be the Zariski decomposition of $L$. Since $P$ is nef and $P \cdot F \leq L \cdot F=0, P$ has vanishing intersection with every $f$-vertical curve. Note that $N$ is an (effective) $f$-vertical curve since $N \cdot F=L \cdot F=0$.

We first show (2). By assumption $N$ must have some components contained in $F_{0}$. Recall that the self-intersection matrix of the components of $N$ is negative-definite. In fact, since $f$-vertical curves in different fibers do not intersect, the same is true just for the components contained in $F_{0}$. Thus, there is an effective curve $G$ supported on $\operatorname{Supp}(N) \cap \operatorname{Supp}\left(F_{0}\right)$ with $0>N \cdot G=L \cdot G$. The same argument shows that in (1) we must have $N=0$ so that $L$ is nef.

The following is a special case of a theorem of [Pet12].

Lemma 2.4 (cf. [Pet12], Theorem 6.8). Let $f: S \rightarrow C$ be a surjective morphism with connected fibers from a smooth projective surface $S$ to a smooth projective curve $C$. Suppose that $L$ is an $f$-numerically trivial nef $\mathbb{R}$-Cartier divisor. Then $L \equiv \alpha F$ for some $\alpha \geq 0$ where $F$ denotes a general fiber of $f$.

Proof. Suppose the theorem fails. There is a divisor $D$ such that $D \cdot L<0$ and $D \cdot F>0$. The latter condition implies that $D$ is $f$-big so that $D+f^{*} H$ 
is pseudo-effective for some ample divisor $H$ on $C$. But $\left(D+m f^{*} H\right) \cdot L<0$, a contradiction.

Corollary 2.5. Let $f: S \rightarrow C$ be a surjective morphism from an irreducible projective surface $S$ to a smooth projective curve $C$ with connected fibers. Suppose that $L$ is a pseudo-effective $\mathbb{R}$-Cartier divisor on $S$ such that $L \cdot C=$ 0 for every $f$-vertical curve $C$. Then $L \equiv f^{*} D$ for some $\mathbb{R}$-Cartier divisor $D$ on $T$.

Proof. When $S$ is smooth this follows from Lemmas 2.3 and 2.4. In general we pass to a resolution $\phi: S^{\prime} \rightarrow S$. Applying the smooth case to $\phi^{*} L$ we find a divisor $D$ such that $\phi^{*} L \equiv(f \circ \phi)^{*} D$. Thus $L \equiv f^{*} D$.

Remark 2.6. Note that Corollary 2.5 shows Theorem 1.2 for surfaces. When combined with Lemma 2.3, it also proves Theorem 1.3 for surfaces: for a pseudo-effective divisor $L$ which is numerically trivial on a general fiber of a surface $S$, Lemma 2.3 shows that the (nef) positive part is trivial on every $f$-vertical curve and is thus a pullback by Corollary 2.5.

2.3. Divisorial Zariski decompositions. We next recall the divisorial Zariski decomposition. This notion was introduced by [Nak04] and [Bou04] as a higher-dimensional analogue of the Zariski decomposition for surfaces.

Definition 2.7. Let $X$ be a smooth projective variety and let $L$ be a pseudoeffective $\mathbb{R}$-Cartier divisor on $X$. Fix an ample divisor $A$ on $X$. Given a prime divisor $\Gamma$ on $X$, we define

$$
\sigma_{\Gamma}(L)=\lim _{\epsilon \rightarrow 0} \min \left\{\operatorname{mult}_{\Gamma}\left(L^{\prime}\right) \mid L^{\prime} \geq 0 \text { and } L^{\prime} \sim_{\mathbb{Q}} L+\epsilon A\right\} .
$$

This definition is independent of the choice of $A$.

[Nak04] shows that for any pseudo-effective divisor $L$ there are only finitely many prime divisors $\Gamma$ with $\sigma_{\Gamma}(L)>0$. Thus we can make the following definition.

Definition 2.8. Let $X$ be a smooth projective variety and let $L$ be a pseudoeffective $\mathbb{R}$-Cartier divisor. We define:

$$
N_{\sigma}(L)=\sum \sigma_{\Gamma}(L) \Gamma \quad P_{\sigma}(L)=L-N_{\sigma}(L)
$$

The decomposition $L=P_{\sigma}(L)+N_{\sigma}(L)$ is called the divisorial Zariski decomposition of $L$.

We need the following properties of the divisorial Zariski decomposition.

Lemma 2.9 ([Nak04], III.1.11 Corollary, III.2.7 Lemma, III.2.5 Lemma). Let $X$ be a smooth projective variety and let $L$ be a pseudo-effective $\mathbb{R}$-Cartier divisor. Then

(1) $N_{\sigma}(L)$ is a finite sum of effective divisors.

(2) For any prime divisor $\Gamma$ of $X$, the restriction $\left.P_{\sigma}(L)\right|_{\Gamma}$ is pseudoeffective. 
(3) If $\phi: Y \rightarrow X$ is a birational map from a smooth projective variety $Y$, then $N_{\sigma}\left(\phi^{*} L\right) \geq \phi^{*} N_{\sigma}(L)$.

The following is a numerical analogue of [Nak04] III.5.2 Lemma.

Lemma 2.10 ([Leh14], Lemma 4.4). Let $f: X \rightarrow Z$ be a surjective morphism from a smooth projective variety to a normal projective variety with connected fibers. Suppose that $L$ is a pseudo-effective $\mathbb{R}$-Cartier divisor such that $\left.L\right|_{F} \equiv 0$ on the general fiber $F$ of $f$. If $\Theta$ is a prime divisor on $Z$ such that $\left.L\right|_{F} \not \equiv 0$ for a general fiber $F$ over $\Theta$, then there is some prime divisor $\Gamma$ on $X$ such that $f(\Gamma)=\Theta$ and $\left.L\right|_{\Gamma}$ is not pseudo-effective.

Proof. The surface case is Lemma 2.3 (2). The general case is proved by cutting down by general very ample divisors on $X$ and $Z$ to reduce to the surface case using Bertini's theorem.

Corollary 2.11. Let $f: X \rightarrow Z$ be a surjective morphism from a smooth projective variety to a normal projective variety with connected fibers. Suppose that $L$ is a pseudo-effective $\mathbb{R}$-Cartier divisor such that $\left.L\right|_{F} \equiv 0$ on the general fiber $F$ of $f$. Then there is a subset $V \subset Z$ that is a countable union of closed sets of codimension 2 such that $\left.P_{\sigma}(L)\right|_{F} \equiv 0$ for every fiber $F$ not lying above $V$.

Proof. Since $L \geq P_{\sigma}(L)$, we see that $\left.P_{\sigma}(L)\right|_{F} \equiv 0$ for a general fiber $F$ of $f$. The conclusion follows from Lemma 2.10 combined with Lemma 2.9 (2).

\subsection{Exceptional divisors.}

Definition 2.12. Let $f: X \rightarrow Z$ be a surjective morphism of integral projective varieties. An $\mathbb{R}$-Cartier divisor $E$ on $X$ is

- $f$-vertical if no component of $\operatorname{Supp}(E)$ dominates $Z$.

- $f$-horizontal otherwise.

We next identify two different ways a divisor can be "exceptional" for a morphism.

Definition 2.13. Let $f: X \rightarrow Z$ be a surjective morphism of integral projective varieties. An $f$-vertical $\mathbb{R}$-Cartier divisor $E$ on $X$ is

- $f$-exceptional if every component $E_{i}$ of $\operatorname{Supp}(E)$ satisfies

$$
\operatorname{codim} f\left(E_{i}\right) \geq 2 \text {. }
$$

- $f$-degenerate if for every prime divisor $\Theta \subset Z$ there is a prime divisor $\Gamma \subset X$ with $f(\Gamma)=\Theta$ and $\Gamma \not \subset \operatorname{Supp}(E)$.

Note that every $f$-exceptional divisor is also $f$-degenerate since any prime divisor $\Theta \subset Z$ is surjected onto by a prime divisor $\Gamma \subset X$ which is (by necessity) not $f$-exceptional.

Although [Nak04] uses a different notion, the arguments of [Nak04] III.5.8 can be applied verbatim to our situation to prove the following lemma. 
Lemma 2.14 ([Nak04], III.5.8 Lemma). Let $f: X \rightarrow Z$ be a surjective morphism of smooth projective varieties with connected fibers. Suppose that $L$ is an effective $f$-vertical $\mathbb{R}$-Cartier divisor. There is an effective $\mathbb{R}$-Cartier divisor $D$ on $Z$ and an effective $f$-exceptional divisor $E$ on $X$ such that

$$
L+E=f^{*} D+F
$$

where $F$ is an effective $f$-degenerate divisor.

As demonstrated by Nakayama, the divisorial Zariski decomposition gives a useful language for understanding $f$-degenerate divisors.

Lemma 2.15 ([GL13], Lemma 2.16). Let $f: X \rightarrow Z$ be a surjective morphism from a smooth projective variety to a normal projective variety and let $D$ be an effective $f$-degenerate $\mathbb{R}$-Cartier divisor. For any pseudo-effective $\mathbb{R}$-Cartier divisor $L$ on $Z$ we have $D \leq N_{\sigma}\left(f^{*} L+D\right)$.

\section{Generically Finite Maps}

In this section we study the behavior of divisors over generically finite morphisms. Such morphisms are a composition of a birational map and a finite map and can be understood by addressing each separately. The following lemma is a well-known consequence of the Negativity of Contraction lemma.

Lemma 3.1. Let $f: X \rightarrow Z$ be a birational morphism from an integral projective variety $X$ to a $\mathbb{Q}$-factorial normal projective variety $Z$. Suppose that $L$ is an $\mathbb{R}$-Cartier divisor on $X$ such that $L$ is $f$-numerically trivial. Then $L \equiv f^{*} D$ for some $\mathbb{R}$-Cartier divisor $D$ on $Z$.

Proof. We may first precompose $f$ by a resolution to assume that $X$ is smooth. Set $D=f_{*} L$. Define the exceptional divisor $E=L-f^{*} f_{*} L$ and write $E=E_{+}-E_{-}$where $E_{+}$and $E_{-}$are effective exceptional divisors with no common components. Note that $N_{\sigma}\left(f^{*} D+E_{-} ; X / Z\right)=E_{-}$. By [Nak04] III.4.2.(2) Lemma we have $N_{\sigma}(L ; X / Z)=0$. Since $N_{\sigma}\left(L+E_{+} ; X / Z\right)=$ $N_{\sigma}\left(f^{*} D+E_{-} ; X / Z\right)$, by [Nak04] III.4.2.(1) Lemma we must have $E_{+}=E_{-}$, a contradiction to the assumption on the components.

Lemma 3.2. Let $f: X \rightarrow Z$ be a surjective finite morphism of normal projective varieties and let $L$ be an $\mathbb{R}$-Cartier divisor on $X$. Let $\left\{T_{i}\right\}_{i=1}^{k}$ be a collection of irreducible curves on $X$. Suppose that there are constants $\alpha_{i}$ such that

$$
L \cdot C=\left(\left.\operatorname{deg} f\right|_{C}\right) \alpha_{i}
$$

for every curve $C$ on $X$ with $f(C)=f\left(T_{i}\right)$. Then there is an $\mathbb{R}$-Cartier divisor $D$ on $Z$ such that $L \cdot T_{i}=f^{*} D \cdot T_{i}$ for every $i$. In particular, if the numerical classes of the $T_{i}$ span $N_{1}(X)$ then $L \equiv f^{*} D$. 
Proof. Let $h: W \rightarrow Z$ denote the Galois closure of $f$ with Galois group $G$. We let $p: W \rightarrow X$ denote the map to $X$. We first show that the $\mathbb{R}$-Cartier divisor

$$
L_{G}:=\frac{1}{|G|} \sum_{g \in G} g\left(p^{*} L\right)
$$

is numerically equivalent to $h^{*} D$ for some $\mathbb{R}$-Cartier divisor $D$ on $Z$. Since $L_{G}$ is $\mathbb{R}$-Cartier, we can write it as a finite sum $L_{G}=\sum a_{i} L_{i}$ for Cartier divisors $L_{i}$ and for $a_{i} \in \mathbb{R}$. For a positive integer $m$, let $\widetilde{L}_{m, i}=\sum_{g \in G} g\left(m L_{i}\right)$. Using [KKV89, 4.2 Proposition], one sees that the natural map $\operatorname{Pic}(Z) \rightarrow$ $\operatorname{Pic}^{G}(W)$ has torsion cokernel. Thus since $\widetilde{L}_{m, i}$ is $G$-invariant, we can find a Cartier divisor $D_{m, i}$ on $Z$ such that $h^{*} D_{m, i} \equiv \widetilde{L}_{m, i}$. Note that the numerical classes of $\frac{1}{m|G|} D_{m, i}$ converge; choose $D_{i}$ to be an $\mathbb{R}$-Cartier divisor representing this class. Set $D=\sum a_{i} D_{i}$. Then

$$
h^{*} D \equiv \lim _{m \rightarrow \infty} \sum a_{i} \frac{1}{m|G|} \widetilde{L}_{m, i}=L_{G} .
$$

Note that if $C$ is a curve on $W$ such that $p(C)=T_{i}$ then $L_{G} \cdot C=p^{*} L \cdot C$ by the assumption on the intersection numbers of $L$. Thus $L \cdot T_{i}=f^{*} D \cdot T_{i}$ for each $i$.

Lemma 3.3. Let $f: X \rightarrow Z$ be a surjective generically finite map from a smooth projective variety $X$ to a $\mathbb{Q}$-factorial normal projective variety $Z$. Let $L$ be an $\mathbb{R}$-Cartier divisor on $X$ and let $\left\{T_{i}\right\}_{i=1}^{k}$ be a collection of irreducible curves on $X$. Suppose that there are constants $\alpha_{i}$ with

$$
L \cdot C=\left(\left.\operatorname{deg} f\right|_{C}\right) \alpha_{i}
$$

for every curve $C$ on $X$ with $f(C)=f\left(T_{i}\right)$.

(1) Suppose that for each $i$ the image $f\left(T_{i}\right)$ is a curve lying in the open locus on $Z$ over which $f$ is flat. Then there is an $\mathbb{R}$-Cartier divisor $D$ on $Z$ such that $L \cdot T_{i}=f^{*} D \cdot T_{i}$ for every $i$.

(2) Suppose that the numerical classes of the $T_{i}$ span $N_{1}(X)$ and that $L \cdot C=0$ for every $f$-vertical curve $C$. Then $L \equiv f^{*} D$ for some $\mathbb{R}$-Cartier divisor $D$ on $Z$.

Proof. Choose a birational model $\phi: \widetilde{X} \rightarrow X$ and a normal birational model $\mu: Z^{\prime} \rightarrow Z$ so that we have a morphism $\tilde{f}: \widetilde{X} \rightarrow Z^{\prime}$ flattening $f$. We may assume that $\phi$ and $\mu$ are isomorphisms on the locus over which $f$ is flat. Let $\psi: X^{\prime} \rightarrow X$ denote a precomposition of $\phi$ with a normalization and let $f^{\prime}: X^{\prime} \rightarrow Z^{\prime}$ denote the natural map. For each $i$ choose an irreducible curve $T_{i}^{\prime}$ on $X^{\prime}$ lying above $T_{i}$. 
We first prove (1). Suppose that $C$ is a curve on $X^{\prime}$ such that $f^{\prime}(C)=$ $f^{\prime}\left(T_{i}^{\prime}\right)$. Since $f\left(\psi\left(T_{i}^{\prime}\right)\right)$ is a curve,

$$
\begin{aligned}
\psi^{*} L \cdot C & =\left(\left.\operatorname{deg} \psi\right|_{C}\right)\left(\left.\operatorname{deg} f\right|_{\psi(C)}\right) \alpha_{i} \\
& =\left(\left.\operatorname{deg} f^{\prime}\right|_{C}\right)\left(\left.\operatorname{deg} \mu\right|_{f^{\prime}(C)}\right) \alpha_{i} \\
& =\left(\left.\operatorname{deg} f^{\prime}\right|_{C}\right)\left(\left.\operatorname{deg} \mu\right|_{f^{\prime}\left(T_{i}^{\prime}\right)}\right) \alpha_{i} .
\end{aligned}
$$

Set $\alpha_{i}^{\prime}=\left(\left.\operatorname{deg} \mu\right|_{f^{\prime}\left(T_{i}^{\prime}\right)}\right) \alpha_{i}$. The set of curves $\left\{T_{i}^{\prime}\right\}$ and the divisor $\psi^{*} L$ satisfy the hypotheses of Lemma 3.2 for the finite map $f^{\prime}$ and the constants $\alpha_{i}^{\prime}$. Lemma 3.2 yields a divisor $D_{Z^{\prime}}$ on $Z^{\prime}$ such that $\psi^{*} L \cdot T_{i}^{\prime}=f^{\prime *} D_{Z^{\prime}} \cdot T_{i}^{\prime}$ for every $i$. Since the $T_{i}$ lie over the locus on which $f$ is flat, $f^{\prime}\left(T_{i}^{\prime}\right)$ avoids the $\mu$-exceptional locus. Thus, setting $D=\mu_{*} D_{Z^{\prime}}$, we obtain $L \cdot T_{i}=f^{*} D \cdot T_{i}$ for every $i$.

We next prove (2), using the same construction as in (1). Applying Lemma 3.1 to $\psi$, we can find finitely many irreducible $\psi$-vertical curves $\left\{S_{j}^{\prime}\right\}_{j=1}^{r}$ whose classes span the kernel of $\psi_{*}$. Together the numerical classes of the $T_{i}^{\prime}$ and $S_{j}^{\prime}$ span all of $N_{1}\left(X^{\prime}\right)$, since the span contains the kernel of $\psi_{*}$ and surjects onto $N_{1}(X)$ via $\psi_{*}$.

Suppose that $C$ is a curve on $X^{\prime}$ such that $f^{\prime}(C)=f^{\prime}\left(T_{i}^{\prime}\right)$. If $f\left(\psi\left(T_{i}^{\prime}\right)\right)$ is a curve, then as before set $\alpha_{i}^{\prime}=\left(\left.\operatorname{deg} \mu\right|_{f^{\prime}\left(T_{i}^{\prime}\right)}\right) \alpha_{i}$. If $f\left(\psi\left(T_{i}^{\prime}\right)\right)$ is a point, then $C$ is also $\left(\mu \circ f^{\prime}\right)$-vertical. Since $\psi^{*} L$ has vanishing intersection with every $\mu \circ f^{\prime}$-vertical curve, $\psi^{*} L \cdot C=0=\psi^{*} L \cdot T_{i}^{\prime}$. Set $\alpha_{i}^{\prime}=0$. Similarly, set $\beta_{j}^{\prime}=0$ for every $S_{j}^{\prime}$.

The set of curves $\left\{T_{i}^{\prime}\right\} \cup\left\{S_{j}^{\prime}\right\}$ and the divisor $\psi^{*} L$ satisfy the hypotheses of Lemma 3.2 for the finite map $f^{\prime}$ and the constants $\alpha_{i}^{\prime}, \beta_{j}^{\prime}$. The result of the lemma indicates that there is a divisor $D_{Z^{\prime}}$ on $Z^{\prime}$ so that $f^{\prime *} D_{Z^{\prime}} \equiv \psi^{*} L$. Since $D_{Z^{\prime}}$ is $\mu$-numerically trivial, Lemma 3.1 yields a divisor $D$ on $Z$ such that $\mu^{*} D \equiv D_{Z^{\prime}}$. Thus $f^{*} D \equiv L$.

\section{Numerical Triviality on Every Fiber}

In this section we give the proof of Theorem 1.2. We start by recalling an example demonstrating that the pseudo-effectiveness of $L$ is necessary in order to have any hope of relating $L$ to divisors on the base.

Example 4.1. Let $E$ be an elliptic curve without complex multiplication and consider the surface $S=E \times E$ with first projection $\pi: S \rightarrow E$. Recall that $N^{1}(S)$ is generated by the fibers $F_{1}, F_{2}$ of the two projections and the diagonal $\Delta$ (see [Laz04] Section 1.5.B). In particular, the subspace of divisors with vanishing intersection against the fibers of $\pi$ is generated by $F_{1}$ and $\Delta-F_{2}$. Since this space has larger dimension than $N^{1}(E)$, most such divisors will not be numerically equivalent to pull-backs from $E$.

The first step in the proof is to show that numerical equivalence of divisors can be detected against curves which are intersections of very ample divisors. 
Proposition 4.2. Suppose that $X$ is an integral projective variety of dimension n. Fix a set of ample Cartier divisors $\mathcal{H}=\left\{H_{1}, \ldots, H_{r}\right\}$ whose numerical classes span $N^{1}(X)$. Then the intersection products $\mathcal{H}^{n-1}$ span $N_{1}(X)$.

Proof. Using results of Matsusaka, [Ful84, Example 19.3.3] shows that a Cartier divisor $D$ is numerically trivial if and only if for a fixed ample divisor $H$ we have that $D \cdot H^{n-1}=D^{2} \cdot H^{n-2}=0$. Writing $D$ and $H$ as linear combinations of elements of $\mathcal{H}$, we obtain the statement.

We now turn to the proof of Theorem 1.2.

Proof of 1.2: The reverse implication is obvious, so we focus on the forward implication.

Suppose that $\phi: X^{\prime} \rightarrow X$ is a resolution of $X$. Note that $\phi^{*} L$ is $(f \circ \phi)$ numerically trivial. If we can find a $D$ so that $\phi^{*} L \equiv(f \circ \phi)^{*} D$ then also $L \equiv f^{*} D$. Thus we may assume that $X$ is smooth.

We next show that for a curve $R$ running through a very general point of $Z$ there is some constant $\alpha_{R}$ such that

$$
L \cdot C=\left(\left.\operatorname{deg} f\right|_{C}\right) \alpha_{R}
$$

for every curve $C$ on $X$ with $f(C)=R$. Let $R^{\prime}$ denote the normalization of $R$ and consider the normalization $Y$ of the unique component of $X \times_{Z} R^{\prime}$ which dominates $R^{\prime}$. Since $R$ goes through a very general point of $Z$ we may assume that the pullback of $L$ to every component of $Y$ is pseudoeffective and that only one component of $Y$ dominates $R^{\prime}$. Consider two curves $C$ and $C^{\prime}$ on $Y$ with $f(C)=f\left(C^{\prime}\right)=R^{\prime}$. By cutting down $Y$ by very ample divisors, we can find a chain of normal surfaces $S_{i}$ connecting $C$ to $C^{\prime}$, all of which map surjectively to $R^{\prime}$ under $f$. We may ensure that $\left.L\right|_{S_{i}}$ is pseudo-effective for every $i$.

Applying Corollary 2.5 to the surface $S_{i}$, we see that there is some divisor $D_{i}$ on $R^{\prime}$ such that $\left.L\right|_{S_{i}} \equiv f^{*} D_{i}$. For $i \geq 1$ let $C_{i}^{\prime}$ denote the curve $S_{i} \cap S_{i+1}$. Since $C_{i}^{\prime}$ dominates $R^{\prime}$, we have

$$
\operatorname{deg}\left(D_{i}\right) \operatorname{deg}\left(\left.f\right|_{C_{i}^{\prime}}\right)=L \cdot C_{i}^{\prime}=\operatorname{deg}\left(D_{i+1}\right) \operatorname{deg}\left(\left.f\right|_{C_{i}^{\prime}}\right) .
$$

Thus there is one fixed $D_{1}$ so that $\left.L\right|_{S_{i}} \equiv f^{*} D_{1}$ for every $i$. Fixing $C$ and letting $C^{\prime}$ vary, we see that the constant $\alpha_{R}=\operatorname{deg}\left(D_{1}\right)$ satisfies the desired condition for every curve above $R$.

Let $W \subset X$ denote a smooth very general intersection of very ample divisors such that the map $f: W \rightarrow Z$ is generically finite and $\left.L\right|_{W}$ is pseudoeffective. Certainly $\left.L\right|_{W}$ has vanishing intersection with any $f$-vertical curve on $W$. Furthermore, Proposition 4.2 shows that we can choose a finite collection of curves $T_{i}$ through very general points whose numerical classes span $N_{1}(W)$. In particular $(\dagger)$ holds over the $T_{i}$. Lemma $3.3(2)$ yields a divisor $D$ on $Z$ such that $\left.L\right|_{W} \equiv f^{*} D$. $D$ is pseudo-effective since it is proportional to the class of $f_{*} L$. 
Apply Proposition 4.2 to $X$ to find a collection of irreducible curves $C_{i}$ on $X$ that are not $f$-vertical and whose numerical classes span $N_{1}(X)$. For each $i$ choose an irreducible curve $C_{i}^{W}$ on $W$ such that $f\left(C_{i}\right)=f\left(C_{i}^{W}\right)$. Since

$$
\begin{aligned}
L \cdot C_{i} & =\left(\left.\operatorname{deg} f\right|_{C_{i}}\right) \alpha_{f\left(C_{i}\right)} \\
& =\left(\left.\operatorname{deg} f\right|_{C_{i}}\right) \frac{L \cdot C_{i}^{W}}{\left.\operatorname{deg} f\right|_{C_{i}^{W}}} \\
& =\left(f^{*} D \cdot C_{i}^{W}\right) \frac{\left.\operatorname{deg} f\right|_{C_{i}}}{\left.\operatorname{deg} f\right|_{C_{i}^{W}}} \\
& =f^{*} D \cdot C_{i}
\end{aligned}
$$

we see that $L \equiv f^{*} D$.

Remark 4.3. There is also a version of Theorem 1.2 for normal varieties. Following [Nak04], we say that an $\mathbb{R}$-Weil divisor $D$ on a normal variety $Z$ is numerically $\mathbb{Q}$-Cartier if there is a smooth birational model $f: X \rightarrow Z$ and an $\mathbb{R}$-Cartier divisor $\widetilde{D}$ such that $\widetilde{D}$ is $f$-numerically trivial and $f_{*} \widetilde{D}=D$.

Suppose that $f: X \rightarrow Z$ is a surjective morphism of normal varieties with connected fibers. Then an $\mathbb{R}$-Cartier divisor $L$ on $X$ is $f$-numerically trivial if and only if there is an $\mathbb{R}$-Weil divisor $D$ on $Z$ such that $D$ is numerically $\mathbb{Q}$-Cartier and $f^{\circledast} D \equiv L$ where $f^{\circledast}$ is the numerical pullback of [Nak04].

The proof runs as follows. First note that an analogue of Lemma 3.1 holds for normal varieties if one only requires $D$ to be a numerically $\mathbb{Q}$-Cartier divisor and replaces $f^{*}$ by $f^{\circledast}$. Indeed, by blowing up we may assume that $X$ also carries a divisor $\widetilde{D}$ as in the definition of numerically $\mathbb{Q}$-Cartier, and then the argument goes through without change. The rest of the proof is exactly the same.

\section{Numerical Triviality on a General Fiber}

In this section we prove Theorem 1.3 in a more general context. The following examples show that Theorem 1.3 is optimal in some sense.

Example 5.1. Let $f: S \rightarrow C$ be a morphism from a smooth surface to a smooth curve. Suppose that $L$ is an effective $f$-degenerate divisor. Then $L$ is not numerically equivalent to the pull-back of a divisor on the base. This is still true on higher birational models of $f$. One must pass to the positive part $P_{\sigma}(L)=0$.

Example 5.2. Let $D$ be a big divisor on a smooth variety $X$ and let $\phi$ : $Y \rightarrow X$ be a blow-up along a smooth center $W$ along which $\sigma_{W}(D)>0$ in the sense of [Nak04] III.2.2 Definition. Then $P_{\sigma}\left(\phi^{*} D\right)<\phi^{*} P_{\sigma}(D)$ is not numerically equivalent to a pull-back of a divisor on $X$. One must pass to the flattening $i d: Y \rightarrow Y$.

The following is a stronger version of Theorem 1.3. 
Theorem 5.3. Let $f: X \rightarrow Z$ be a surjective morphism with connected fibers from a smooth projective variety $X$ to a normal projective variety $Z$. Suppose that $L$ is a pseudo-effective $\mathbb{R}$-Cartier divisor on $X$ such that $P_{\sigma}(L)_{F} \equiv 0$ for a general fiber $F$ of $f$. Then there is a smooth birational model $\phi: Y \rightarrow X$, a map $g: Y \rightarrow Z^{\prime}$ birationally equivalent to $f$, and an $\mathbb{R}$-Cartier divisor $D$ on $Z^{\prime}$ such that $P_{\sigma}\left(\phi^{*} L\right) \equiv P_{\sigma}\left(g^{*} D\right)$.

Remark 5.4. In particular this theorem may be applied whenever $\nu\left(\left.L\right|_{F}\right)=$ 0 for a general fiber $F$, where $\nu$ denotes the numerical dimension of [Nak04] and [Bou04]. In this situation we have

$$
\left.P_{\sigma}(L)\right|_{F} \leq P_{\sigma}\left(\left.L\right|_{F}\right) \equiv 0
$$

and since $\left.P_{\sigma}(L)\right|_{F}$ is pseudo-effective for a general fiber $F$ by [Pet12, 6.8 Theorem] and the duality theorem of [BDPP13] we have $\left.P_{\sigma}(L)\right|_{F} \equiv 0$.

Proof. There is an integral birational model $\mu: X^{\prime} \rightarrow X$, a smooth birational model $Z^{\prime}$ of $Z$, and a morphism $f^{\prime}: X^{\prime} \rightarrow Z^{\prime}$ flattening $f$. Let $\psi: Y \rightarrow X^{\prime}$ denote a smooth model. We let $g$ denote the composition $f^{\prime} \circ \psi$ and let $\phi$ denote the composition $\mu \circ \psi$. Note that every $g$-exceptional divisor is also $\phi$-exceptional. We still have that $\left.P_{\sigma}\left(\phi^{*} L\right)\right|_{F} \equiv 0$ for a general fiber $F$ of $g$ by the same argument as in Remark 5.4.

By Corollary 2.11, there is a subset $V \subset Z^{\prime}$ that is a countable union of codimension 2 subsets such that $P_{\sigma}\left(\phi^{*} L\right)$ is numerically trivial along every fiber of $g$ not over $V$. We also include in $V$ the codimension $\geq 2$ locus over which $g$ is not flat. In particular, suppose that the curve $R \subset Z^{\prime}$ avoids $V$ and $P_{\sigma}\left(\phi^{*} L\right)$ is pseudo-effective when restricted to the preimage of $R$. By the same argument as in the proof of Theorem 1.2, there is some constant $\alpha_{R}$ such that

$$
P_{\sigma}\left(\phi^{*} L\right) \cdot C=\operatorname{deg}\left(\left.g\right|_{C}\right) \cdot \alpha_{R}
$$

for every curve $C$ with $g(C)=R$.

We next apply the generically finite case to construct a divisor $D_{1}$. Choose a smooth very general intersection $W$ of very ample divisors on the smooth variety $Y$ so that the map $\left.g\right|_{W}: W \rightarrow Z$ is generically finite. By choosing $W$ very general we may assume that the divisor $\left.P_{\sigma}\left(\phi^{*} L\right)\right|_{W}$ is pseudo-effective.

Consider the subspace of $N_{1}(W)$ generated by irreducible curves $C$ that avoid $g^{-1}(V)$ and run through a very general point of $W$. We may choose a finite collection of irreducible curves $\left\{T_{i}\right\}$ satisyfing these two properties whose numerical classes span this subspace. By $\left(^{*}\right)$ there are constants $\alpha_{i}$ so that

$$
\left.P_{\sigma}\left(\phi^{*} L\right)\right|_{W} \cdot C=\operatorname{deg}\left(\left.g\right|_{C}\right) \cdot \alpha_{i}
$$

for every curve $C \subset W$ with $g(C)=g\left(T_{i}\right)$. Applying Lemma 3.3 (1), we find a divisor $D_{1}$ on $Z^{\prime}$ with $P_{\sigma}\left(\phi^{*} L\right) \cdot T_{i}=g^{*} D_{1} \cdot T_{i}$ for every $i$. Since $D_{1}$ is constructed by rescaling a push forward of $P_{\sigma}\left(\phi^{*} L\right)$, it is pseudo-effective. Furthermore $P_{\sigma}\left(\phi^{*} L\right) \cdot C=g^{*} D_{1} \cdot C$ for any curve $C$ through a very general 
point of $W$ such that $g(C)$ avoids $V$, since $C$ is numerically equivalent to a sum of the $T_{i}$.

We next relate $P_{\sigma}\left(\phi^{*} L\right)$ and $g^{*} D_{1}$. Since $f^{\prime}$ is flat and $\mu$ is birational, $\mu\left(f^{\prime-1} V\right)$ is a countable union of codimension $\geq 2$ subvarieties in $X$. By Proposition 4.2 we may choose curves $S_{i}^{X}$ avoiding this locus and running through a very general point of $X$ whose numerical classes form a basis for $N_{1}(X)$. Let $\left\{S_{i}\right\}$ consist of the strict transforms of these curves on $Y$. Since the $S_{i}$ are generic, for each we may choose a curve $S_{i}^{W} \subset W$ going through a very general point and such that $g\left(S_{i}^{W}\right)=g\left(S_{i}\right)$ avoids $V$. This guarantees that $L \cdot S_{i}^{W}=g^{*} D_{1} \cdot S_{i}^{W}$. By construction $P_{\sigma}\left(\phi^{*} L\right) \cdot S_{i}^{W}$ and $P_{\sigma}\left(\phi^{*} L\right) \cdot S_{i}$ can be compared using $(*)$. Arguing as in the proof of Theorem 1.2, we see that $P_{\sigma}\left(\phi^{*} L\right) \cdot S_{i}=g^{*} D_{1} \cdot S_{i}$ for every $i$. This proves that

$$
\phi_{*} P_{\sigma}\left(\phi^{*} L\right) \equiv \phi_{*} g^{*} D_{1} \text {. }
$$

Choose effective $\phi$-exceptional divisors $E$ and $F$ with no common components such that

$$
P_{\sigma}\left(\phi^{*} L\right)+E \equiv g^{*} D_{1}+F .
$$

Note that since $f: X \rightarrow Z$ is generically flat, no $\phi$-exceptional divisor dominates $Z^{\prime}$. In particular $F$ is $g$-vertical, so we may apply Lemma 2.14 to $F$ to find

$$
F=g^{*} D_{2}+F_{\text {deg }}-F_{\text {exc }}
$$

where $D_{2}$ is effective, $F_{\text {deg }}$ is $g$-degenerate, and $F_{\text {exc }}$ is $g$-exceptional. Set $D=D_{1}+D_{2}$. Then

$$
P_{\sigma}\left(\phi^{*} L\right)+E+F_{\text {exc }} \equiv g^{*} D+F_{\text {deg }} .
$$

Since $F_{\text {deg }}$ is $g$-degenerate, Lemma 2.15 shows $P_{\sigma}\left(g^{*} D+F_{\text {deg }}\right)=P_{\sigma}\left(g^{*} D\right)$. Similarly, since $\left(E+F_{\text {exc }}\right)$ is $\phi$-exceptional,

$$
\begin{aligned}
P_{\sigma}\left(\phi^{*} L\right) & \leq P_{\sigma}\left(P_{\sigma}\left(\phi^{*} L\right)+E+F_{\text {exc }}\right) \\
& \leq P_{\sigma}\left(\phi^{*} L+E+F_{\text {exc }}\right) \\
& =P_{\sigma}\left(\phi^{*} L\right) \text { by Lemma } 2.15 .
\end{aligned}
$$

Taking the positive part of both sides of $\left({ }^{* *}\right)$ yields $P_{\sigma}\left(\phi^{*} L\right) \equiv P_{\sigma}\left(g^{*} D\right)$.

\section{Numerical Dimension AND PULlBaCK}

The numerical dimension is an important invariant of a divisor defined by [Nak04] and [Bou04]. There are many natural ways to define the numerical dimension; we will use the following two.

Definition 6.1 ([Leh13] Theorem 1.1). Let $X$ be a smooth complex projective variety and let $L$ be a pseudo-effective $\mathbb{R}$-Cartier divisor on $X$. Then the two following quantities coincide:

(1) $\max \left\{k \in \mathbb{Z}_{\geq 0} \mid \lim \sup _{m \rightarrow \infty} \frac{h^{0}\left(X, \mathcal{O}_{X}(\lfloor m L\rfloor+A)\right)}{m^{k}}>0\right\}$ where $A$ is a fixed sufficiently ample Cartier divisor on $X$. 
(2) $\max \left\{\operatorname{dim} W \mid \inf _{\phi: Y \rightarrow X} \operatorname{vol}_{\widetilde{W}}\left(\left.P_{\sigma}\left(\phi^{*} L\right)\right|_{\widetilde{W}}\right)>0\right\}$ where $W$ varies over all complete intersections of very general elements of a very ample linear series, $\phi$ varies over all birational maps such that no exceptional center contains $W$ and $\widetilde{W}$ denotes the strict transform of $W$.

We denote this common quantity by $\nu(L)$. (While Definition $(2)$ is slightly different from [Leh13] Theorem 1.1.(4), the equivalence is shown by [Leh13] Proposition 4.21.)

The numerical dimension is preserved by birational pullback. Thus, we define the numerical dimension for a pseudo-effective $\mathbb{R}$-Cartier divisor on any integral complex projective variety by pulling back to a resolution.

To work with the numerical dimension, we need to recall the definition of the diminished base locus of a divisor.

Definition 6.2. Let $X$ be a smooth complex projective variety and let $L$ be a pseudo-effective $\mathbb{R}$-divisor on $X$. We define the $\mathbb{R}$-stable base locus of $L$ to be the subset of $X$ given by

$$
\mathbf{B}_{\mathbb{R}}(L)=\bigcap\left\{\operatorname{Supp}\left(L^{\prime}\right) \mid L^{\prime} \geq 0 \text { and } L^{\prime} \sim_{\mathbb{R}} L\right\} .
$$

The diminished base locus of $L$ is then defined to be

$$
\mathbf{B}_{-}(L)=\bigcup_{A \text { ample } \mathbb{R} \text {-divisor }} \mathbf{B}_{\mathbb{R}}(L+A) .
$$

The diminished base locus is a countable union of proper subvarieties of $X$, but need not be closed (see [Les14]).

Remark 6.3. Fix a very ample linear series $|H|$ on $X$. For a very general complete intersection $W$ of members of $|H|$, we can ensure that for any component $B \subset \mathbf{B}_{-}(L)$ the codimension of $B \cap W$ in $W$ is at least the codimension of $B$ in $X$. Furthermore, we can ensure the same is true for $\mathbf{B}_{-}\left(\phi^{*} L\right)$ and the strict transform $\widetilde{W}$ where $\phi$ is any birational map $\phi: Y \rightarrow$ $X$ such that no exceptional center is contained in $W$.

Proof of Proposition 1.4: Let $n$ denote the dimension of $X$ and let $d$ denote the dimension of $Z$. By passing to a birational model of $f$ we may suppose that $X$ and $Z$ are smooth. The case when $f$ is generically finite is proved in [Leh13] Proposition 4.20 (using Definition 5 of [Leh13] Theorem 1.1). In general, since we can prove the statement for each portion of the Stein factorization separately, we can reduce to the case when $f$ has connected fibers and $X$ and $Z$ are smooth.

We first show that $\nu(D) \leq \nu\left(f^{*} D\right)$. Indeed, fix an ample Cartier divisor $A$ on $Z$ and choose an ample Cartier divisor $H$ on $X$ such that $H-\phi^{*} A$ is very ample. Then the inequality is clear from definition (1) of $\nu$.

Conversely, let the very ample linear series $|H|$ on $X$ and the very general intersection $W$ of elements of $H$ be a choice of data which attains the maximum as in definition (2) of $\nu$ so that $\operatorname{dim}(W)=k=\nu\left(f^{*} D\right)$. For 
notational convenience we set $L=f^{*} D$. By Remark 6.3, for any birational map $\phi: Y \rightarrow X$ such that no exceptional center is contained in $W$

$$
\left.P_{\sigma}\left(\phi^{*} L\right)\right|_{\widetilde{W}} \leq\left.\left.\phi\right|_{\widetilde{W}} ^{*} L\right|_{W}
$$

Since the volume of the divisor on the left is positive, the volume on the right is as well. In particular, we see that $\operatorname{dim} W \leq \operatorname{dim} Z$.

Note that $W$ is the base locus of a linear series $V \subset|H|$ of dimension $n-k$ and that $k \leq d$. Fix $n-d$ very general elements of $V$ and set $T$ to be their intersection. Consider varying $\phi: Y \rightarrow X$ over all birational maps whose exceptional centers are not contained in $T$. The strict transforms $\widetilde{T}$ of $T$ then vary over all birational models of $T$. By Remark 6.3, for each such map we have

$$
\left.P_{\sigma}\left(\phi^{*} L\right)\right|_{\widetilde{T}} \leq\left.\left.\phi\right|_{\widetilde{T}} ^{*} L\right|_{T}
$$

Furthermore, Remark 6.3 also shows that the divisor on the left is movable, since every component of $\mathbf{B}_{-}\left(P_{\sigma}\left(\phi^{*} L\right)\right) \cap T$ has codimension at least 2 in $T$. Thus

$$
\left.P_{\sigma}\left(\phi^{*} L\right)\right|_{\widetilde{T}} \leq P_{\sigma}\left(\left.\left.\phi\right|_{\widetilde{T}} ^{*} L\right|_{T}\right) .
$$

If $\left.\phi\right|_{\widetilde{T}}$ has no exceptional centers contained in $W$, then by restricting to $\widetilde{W}$, we find

$$
\operatorname{vol}\left(\left.P_{\sigma}\left(\phi^{*} L\right)\right|_{\widetilde{W}}\right) \leq \operatorname{vol}\left(\left.P_{\sigma}\left(\left.\left.\phi\right|_{\widetilde{T}} ^{*} L\right|_{T}\right)\right|_{\widetilde{W}}\right) .
$$

Using definition (2) of $\nu$, we conclude that $\nu\left(\left.f^{*} D\right|_{T}\right) \geq \nu\left(f^{*} D\right)$. Since $\left.f\right|_{T}$ is generically finite, [Leh13] Proposition 4.20 shows that $\nu\left(\left.f^{*} D\right|_{T}\right)=\nu(D)$. This proves the reverse inequality.

\section{REFERENCES}

$\left[\mathrm{BCE}^{+} 02\right]$ T. Bauer, F. Campana, T. Eckl, S. Kebekus, T. Peternell, S. Rams, T. Szemberg, and L. Woltzlas, A reduction map for nef line bundles, Complex Geometry (Göttingen, 2000), Springer, Berlin, 2002, pp. 27-36.

[BDPP13] S. Boucksom, J.P. Demailly, M. Păun, and T. Peternell, The pseudo-effective cone of a compact Kähler manifold and varieties of negative Kodaira dimension, J. Alg. Geom. 22 (2013), no. 2, 201-248.

[Bou04] S. Boucksom, Divisorial Zariski decompositions on compact complex manifolds, Ann. Sci. École Norm. Sup. 37 (2004), no. 4, 45-76.

[DJV13] Olivier Debarre, Zhi Jiang, and Claire Voisin, Pseudo-effective classes and pushforwards, Pure and Applied Mathematics Quarterly 9 (2013), no. 4, 643664 .

[Eck05] T. Eckl, Numerically trival foliations, Iitaka fibrations, and the numerical dimension, 2005, arXiv:math/0508340v1.

[Fuj79] T. Fujita, On Zariski problem, Proc. Japan Acad. Ser. A Math. Sci. 55 (1979), no. 3, 106-110.

[Ful84] William Fulton, Intersection theory, Ergebnisse der Mathematik und ihrer Grenzgebiete (3) [Results in Mathematics and Related Areas (3)], vol. 2, Springer-Verlag, Berlin, 1984.

[GL13] Y. Gongyo and B. Lehmann, Reduction maps and minimal model theory, Compos. Math. 149 (2013), no. 2, 295-308. 
[KKV89] Friedrich Knop, Hanspeter Kraft, and Thierry Vust, The Picard group of a Gvariety, Algebraische Transformationsgruppen und Invariantentheorie, DMV Sem., vol. 13, Birkhäuser, Basel, 1989, pp. 77-87.

[Laz04] Robert Lazarsfeld, Positivity in algebraic geometry I-II, Ergebnisse der Mathematik und ihrer Grenzgebiete. 3. Folge, vol. 48-49, Springer-Verlag, Berlin Heidelberg, 2004.

[Leh13] Brian Lehmann, Comparing numerical dimensions, Algebra Number Theory 7 (2013), no. 5, 1065-1100.

[Leh14] $\quad$ On Eckl's pseudo-effective reduction map, Trans. Amer. Math. Soc. 366 (2014), no. 3, 1525-1549.

[Les14] John Lesieutre, The diminished base locus is not always closed, Compos. Math. 150 (2014), no. 10, 1729-1741. MR 3269465

[Nak04] N. Nakayama, Zariski-decomposition and abundance, MSJ Memoirs, vol. 14, Mathematical Society of Japan, Tokyo, 2004.

[Pet12] T. Peternell, Varieties with generically nef tangent bundles, J. Eur. Math. Soc. (JEMS) 14 (2012), no. 2, 571-603.

[Ray72] M. Raynaud, Flat modules in algebraic geometry, Comp. Math. 24 (1972), $11-31$.

[Zar64] O. Zariski, The theorem of Riemann-Roch for high multiples of an effective divisor on an algebraic surface, Ann. of Math. 76 (1964), no. 2, 560-615.

Department of Mathematics, Boston College, Chestnut Hill, MA 02467

E-mail address: blehmann@rice.edu 